\title{
Abstinence from Cocaine Reduces High-Risk Responses on a Gambling Task
}

The inability of cocaine addicts to modify their behavior in the face of adverse consequences and their poor risk assessment resemble the impaired judgment observed in patients with lesions of the ventromedial prefrontal cortex (VmPFC) (Damasio 1985; Bechara et al. 1994; Grant et al. 1997). Patients with VmPFC lesions demonstrate specific deficits involving the inability to make advantageous decisions that involve balancing reward against risk when taking into account future consequences (Damasio 1985). A Gambling Task was developed to quantify this VmPFC deficit (Bechara et al. 1994). A recent study reported that polydrug abusers perform significantly worse on the Gambling Task when compared to controls drawn from the same community (Grant et al. 1997).

On a pilot basis, we evaluated the performance on the Gambling Task in six recently abstinent (more than 4 days) versus six nonabstinent cocaine-dependent (CD) males. All the subjects were outpatients recruited from treatment and research clinics of a metropolitan Department of Veterans Affairs Medical Center. Written informed consent was obtained from all subjects before study participation. Inclusion criteria were: DSM-IV diagnosis for $\mathrm{CD}$, age 33 to 55 , history of $\mathrm{CD}$ longer than 1 year, and use of cocaine of at least $\$ 50 /$ week, primarily by smoking. Subjects were excluded for presence of other psychiatric disorders, dependence on other substances (except nicotine and alcohol), and significant medical or brain diseases. Length of abstinence was assessed by self-report, further validated by urinalysis testing three times per week. The two groups did not statistically differ in age, education, race, and length of exposure to cocaine $(p>.55)$.

The Gambling Task employed in the current study was a computerized version of the task described in detail by Bechara et al. (1994). A net outcome score was computed by subtracting the total number of high-risk selections from low-risk selections. The maximum and minimum scores that can be achieved range from -60 to +60 , with lower scores indicating poorer performance and a preference for high-risk choices, which is characteristic of subjects with VmPFC damage (Bechara et al. 1994; Bechara et al. 1998). Two control cognitive tasks were included to address the issue of specificity related to deficits on the gambling task: the Wisconsin Card Sorting Test (WCST) and the California Verbal Learning Test (CVLT). The WCST assesses concept formation and set shifting and is dependent on the integrity of the dorsolateral prefrontal cortex, and the CVLT assesses verbal learning and short-term memory associated with the functions of the left temporal region (Sullivan et al. 1994).

The individual scores for each subject are displayed in Table 1. Unpaired $t$-test revealed a significant difference in net outcome score between the two groups, with the abstinent group achieving a substantially higher mean $( \pm \mathrm{SD})$ score on the Gambling Task than that of the nonabstinent group $(7.7 \pm 14.7$ vs. $-17.0 \pm 21.3$, respectively; $\mathrm{t}=2.33, p=.04$ ). No significant differences in performance were found between groups on any of the component scores on the WCST and CVLT $(p>.42)$. Cocaine-dependent subjects who were currently using cocaine achieved a negative outcome score on the Gambling Task, suggesting that cocaine use impairs risk assessment and advantageous decision making.

These results have striking face validity given the often described risk-taking behavior and unresponsiveness to increasing evidence of adverse consequences involved in cocaine use. Cocaine-dependent patients with 4 or more days of abstinence seem to be able to evaluate risk better as they achieved a net positive outcome score as compared to the negative score of patients with 
Table 1. Number of Days Abstinent for Each Subject and Individual Net Outcome Scores* for the Gambling Task

\begin{tabular}{lccr}
\hline Abstinent group & Age & Days Abstinent & Score \\
\hline Subject 1 & 55 & 21 & 4 \\
Subject 2 & 38 & 10 & -6 \\
Subject 3 & 35 & 24 & 24 \\
Subject 4 & 34 & 21 & 0 \\
Subject 5 & 33 & 4 & -4 \\
Subject 6 & 37 & 4 & 28 \\
Nonabstinent group & & & \\
Subject 1 & 35 & 2 & -44 \\
Subject 2 & 39 & 2 & -34 \\
Subject 3 & 35 & 1 & -18 \\
Subject 4 & 46 & 3 & -4 \\
Subject 5 & 38 & 3 & 16 \\
Subject 6 & 34 & 1 & \\
\hline
\end{tabular}

* Net outcome score computed by subtracting total number of highrisk selections (Decks $A+B$ ) from low-risk selections (Decks $C+D)$.

fewer than 4 days of abstinence. This suggests that an acute pharmacologic effect of cocaine may be partially responsible for the poor risk assessment of $C D$ patients.

The Gambling Task deficit may be mediated by a specific effect of cocaine on the VmPFC, because performance on cognitive tasks involving other brain regions such as dorsolateral PFC (assessed by WCST) and left medial temporal cortex (assessed by CVLT) were not affected by abstinence. Recent data support the possibility that VmPFC can manifest as a specific deficit in decision making with sparing of cognitive functions performed by other brain regions (Bechara et al. 1998).

These findings highlight the possible usefulness of this task in evaluating decision-making impairments in clinical settings, such as assessing the impact of pharmacologic interventions on pertinent clinical variables. Future investigations should include a larger sample, female subjects, a matching normal control sample, random assignment of subjects, within-subjects design, and assessment of gambling history. An investigation of the association between performance on this task and functional imaging parameters of the VmPFC may also be warranted.

\section{ACKNOWLEDGMENTS}

This work was supported by the Research and Psychiatry Services of the Department of Veterans Affairs, VISIN16 MIRECC, Medication Development Division of the National Institute on Drug Abuse, and the Marie Wilson Howells Endowment.

George Bartzokis, M.D. ${ }^{\mathrm{a}, \mathrm{b}, \mathrm{c}, \mathrm{d}}$

Po H. Lu, M.A. ${ }^{\text {a,c }}$

Mace Beckson, M.D.c,d

Ruth Rapoport, B.S. ${ }^{c}$

Steven Grant, Ph.D. ${ }^{\text {e }}$

Eve J. Wiseman, M.D.a,b

Edythe D. London, Ph.D. ${ }^{\mathrm{e}}$

aDepartment of Psychiatry

University of Arkansas for Medical Sciences

Little Rock, AR

${ }^{b}$ Mental Health Service Line

Central Arkansas Veterans Healthcare System

Little Rock, AR

${ }^{\mathrm{c} G r e a t e r ~ L o s ~ A n g e l e s ~ V A ~ H e a l t h c a r e ~ S y s t e m ~}$

West Los Angeles, CA

${ }^{\mathrm{d}}$ Department of Psychiatry

University of California

Los Angeles, CA

'Brain Imaging Center

National Institute on Drug Abuse

Baltimore, MD

\section{REFERENCES}

Bechara A, Damasio AR, Damasio H, Anderson SW (1994): Insensitivity to future consequences following damage to human prefrontal cortex. Cognition 50:7-15

Bechara A, Damasio H, Tranel D, Anderson SW (1998): Dissociation of working memory from decision making within the human prefrontal cortex. J Neurosci 18:428437

Damasio AR (1985): The Frontal Lobes. In Heilman KM, Valenstein E (eds), Clinical Neuropsychology. New York, Oxford University Press, pp 339-375

Grant SJ, Contoreggi C, London ED (1997): Drug abusers show impaired performance on a test of orbitofrontal function. Proceeding of the Society for Neuroscience Annual Meeting, October 25-30, New Orleans, LA

Sullivan EV, Shear PK, Zipursky RB, Sagar HJ, Pfefferbaum A (1994): A deficit profile of executive, memory, and motor functions in schizophrenia. Biol Psychiat 36:641-653 